\title{
Gravity in the shadow of stable atoms and their three interactions
}

\author{
U. V. S. Seshavatharam ${ }^{1} \&$ S. Lakshminarayana ${ }^{2}$ \\ ${ }^{1}$ Honorary Faculty, I-SERVE, Survey no-42, Hitech city, Hyderabad-84, Telangana, India. \\ ${ }^{2}$ Department of Nuclear Physics, Andhra University, Visakhapatnam-03, AP, India \\ Emails: seshavatharam.uvs@gmail.com (and)sln@auvsp.edu.in \\ Orcid numbers : 0000-0002-1695-6037 (and) 0000-0002-8923-772X
}

\begin{abstract}
Even though materialistic atoms are having independent existence in this current accelerating universe, they are not allowing scientists and engineers to explore the secrets of gravity at atomic scale. This may be due to incomplete unification paradigm, inadequacy of known physics and technological difficulties etc. In this challenging scenario, one fundamental question to be answered is: Is Newtonian gravitational constant having a physical existence? We would like to suggest that, it's a man created empirical constant and is having no physical existence. Clearly speaking, it's not real but virtual. For understanding the secrets of large scale gravitational effects, scientists consider it as a physical constant. In the same way, each atomic interaction can be allowed to have its own gravitational constant. With further study, their magnitudes can be refined for a better understanding of the nature. Thinking in this way, we tried to fit the Newtonian gravitational constant. It's estimated value seems to be $6.679855 \times 10^{\wedge}(-11)$ $\mathrm{m} 3 / \mathrm{kg} / \mathrm{sec} 2$. Proceeding further, the famous radiation constants ( $\hbar c$ and $h c$ ) can be shown to be complex or secondary physical constants. By considering proton neutron stability, nuclear binding energy, nuclear charge radii, neutron life time, Fermi's weak coupling constant and strong coupling constant, we are trying to understand the validity of the proposed three atomic gravitational constants. It needs further study.
\end{abstract}

Keywords: materialistic atoms, three atomic gravitational constants, Newtonian gravitational constant, materialistic quantum gravity.

\section{Introduction}

In this paper, with respect to the available physics literature pertaining to large gravitational coupling constants [1-6] and with reference to the virtual Newtonian gravitational constant, we propose three different gravitational constants assumed to be associated with the observed three atomic interactions. To validate them, we study their possible role in understanding nuclear stability and binding energy [7-12] for light, medium, heavy and super heavy atomic nuclides. The most desirable cases of any unified description are:

a) To implement gravity in microscopic physics and to estimate the magnitude of the Newtonian gravitational constant $\left(G_{N}\right)$.

b) To develop a model of microscopic quantum gravity.

c) To simplify the complicated issues of known physics. (Understanding nuclear stability, nuclear binding energy, nuclear charge radii and neutron life time etc.)

d) To predict new effects, arising from a combination of the fields inherent in the unified description. (Understanding nuclear elementary charge, strong coupling constant, Fermi's weak coupling constant and radiation constants etc.)

In this context, in our recent and earlier publications [13-30], we could present many interesting relations among all the key physical constants of nuclear and atomic physics. Objectives of this paper are:

$>$ To understand the possible role of Newtonian gravitational constant in microscopic physics.

$>$ To see the possibility of estimating the magnitude of Newtonian gravitational constant in a theoretical approach within the scope of nuclear physics.

$>$ To see the possibility of understanding the historical mystery of the radiation constants ( $\hbar c$ and $h c)$.

\section{Four simple assumptions}

With reference to our recent paper publications and conference proceedings [13-30], we propose the following four assumptions.

1) There exist four different gravitational constants associated with gravitational, weak, electromagnetic and strong interactions. Let, Nuclear gravitational constant $=G_{s}$ 
Weak gravitational constant $=G_{w}$

Electromagnetic gravitational constant $=G_{e}$

Newtonian gravitational constant $=G_{N}$

With our long experience in this approach, we present the following 9 relations as "Reference relations". With further study, they can be reviewed for a better understanding.

$$
\left.\begin{array}{c}
\frac{m_{p}}{m_{e}} \cong 2 \pi \sqrt{\frac{4 \pi \varepsilon_{0} G_{e} m_{e}^{2}}{e^{2}}} \\
\hbar c \cong\left(\frac{m_{p}}{m_{e}}\right)^{2}\left(G_{e}^{2} G_{N}\right)^{1 / 3} m_{p}^{2} \\
\cong 16 \pi^{4}\left(\frac{4 \pi \varepsilon_{0} G_{e} m_{e}^{2}}{e^{2}}\right)^{2}\left(G_{e}^{2} G_{N}\right)^{1 / 3} m_{e}^{2}
\end{array}\right\}
$$

Based on relations (1) (2), (3) and (4), magnitudes of $\left(G_{e}, G_{N}, G_{s}, G_{w}\right)$ can be estimated.

2) There exists a strong elementary charge in such a way that,

$$
\begin{gathered}
\frac{e_{s}}{e} \cong\left(\frac{G_{s} m_{p}^{2}}{\hbar c}\right) \cong\left[\frac{G_{s} m_{e}^{2}}{\left(G_{e}^{2} G_{N}\right)^{1 / 3} m_{p}^{2}}\right] \\
\frac{e_{s}^{2}}{e^{2}} \cong\left(\frac{G_{s} m_{p}^{2}}{\hbar c}\right)^{2} \cong\left(\frac{G_{s} m_{p}^{3}}{G_{e} m_{e}^{3}}\right) \\
\frac{e_{s} G_{s}}{e G_{w}} \cong\left(\frac{m_{p}}{m_{e}}\right)^{2}
\end{gathered}
$$

3) Strong coupling constant [31,32] can be expressed with,

$$
\alpha_{s} \cong\left(\frac{e}{e_{s}}\right)^{2} \cong\left(\frac{\hbar c}{G_{s} m_{p}^{2}}\right)^{2} \cong\left(\frac{G_{e} m_{e}^{3}}{G_{s} m_{p}^{3}}\right)
$$

4) Nuclear charge radius can be addressed with,

$$
R_{0} \cong \frac{2 G_{s} m_{p}}{c^{2}}
$$

3. Characteristic relations connected with Fermi's weak coupling constant

Quantitatively, Fermi's weak coupling can be addressed with,

$$
G_{F} \cong\left(\frac{m_{e}}{m_{p}}\right)^{2} \hbar c R_{0}^{2} \cong \frac{4 G_{s}^{2} m_{e}^{2} \hbar}{c^{3}}
$$

Based on the above relations,

$$
\begin{aligned}
& G_{F} \cong\left[\left(G_{e} m_{p}^{2}\right)^{2}\left(G_{N} m_{p}^{2}\right)\right]^{\frac{1}{3}}\left(\frac{2 G_{s} m_{p}}{c^{2}}\right)^{2} \\
& \cong \hbar c\left(\frac{4 G_{w} \hbar}{c^{3}}\right) \cong \frac{4 G_{w} \hbar^{2}}{c^{2}}
\end{aligned}
$$

4. To estimate the magnitudes of

$$
\left(e_{s}, \alpha_{s}, G_{e}, G_{w}, G_{N}, G_{F}\right)
$$

Based on the above relations,

$$
\left\{\begin{array}{l}
G_{e} \cong 2.374335 \times 10^{37} \mathrm{~m}^{3} \mathrm{~kg}^{-1} \mathrm{sec}^{-2} \\
G_{N} \cong 6.679855 \times 10^{-11} \mathrm{~m}^{3} \mathrm{~kg}^{-1} \mathrm{sec}^{-2} \\
G_{s} \cong 3.329561 \times 10^{28} \mathrm{~m}^{3} \mathrm{~kg}^{-1} \mathrm{sec}^{-2} \\
G_{w} \cong 2.909745 \times 10^{22} \mathrm{~m}^{3} \mathrm{~kg}^{-1} \mathrm{sec}^{-2} \\
G_{F} \cong 1.44021 \times 10^{-62} \mathrm{~J} \cdot \mathrm{m}^{3} \\
\alpha_{s} \cong 0.115194 \\
e_{s} \cong 4.720587 \times 10^{-19} \mathrm{C}
\end{array}\right\}
$$

\section{Understanding proton-neutron stability with} three atomic gravitational constants

Let,

$$
\left.\begin{array}{l}
s \cong\left\{\left(\frac{e_{s}}{m_{p}}\right) \div\left(\frac{e}{m_{e}}\right)\right\} \cong 0.001605 \\
\cong \frac{G_{s} m_{p} m_{e}}{\hbar c} \cong \frac{\hbar c}{G_{e} m_{e}^{2}} \cong \frac{G_{s}^{2}}{G_{e} G_{w}}
\end{array}\right\}
$$

Nuclear beta stability line can be addressed with a relation of the form [relation 8 of reference. 9], 


$$
\begin{aligned}
A_{s} & \cong 2 Z+s(2 Z)^{2} \cong 2 Z+(4 s) Z^{2} \\
& \cong 2 Z+k Z^{2} \cong Z(2+k Z)
\end{aligned}
$$$$
\text { where }(4 s) \cong k \cong 0.0064185
$$

By considering a factor like $[2 \pm \sqrt{k}]$, likely possible range of $A_{s}$ can be addressed with,

$$
\begin{array}{r}
\left(A_{s}\right)_{\text {lower }}^{\text {upper }} \cong Z[(2 \pm 0.08)+k Z] \\
\rightarrow\left\{\begin{array}{l}
\left(A_{s}\right)_{\text {lower }} \cong Z(1.92+k Z) \\
\left(A_{s}\right)_{\text {mean }} \cong Z(2.0+k Z) \\
\left(A_{s}\right)_{\text {upper }} \cong Z(2.08+k Z)
\end{array}\right\}
\end{array}
$$

Interesting point to be noted is that, for $\mathrm{Z}=112,113$ and 114, estimated lower stable mass numbers are 296, 299 and 302 respectively. Corresponding neutron numbers are 184, 186 and 188. These neutron numbers are very close to the currently believed shell closure at $\mathrm{N}=184$. It needs further study [33].

\section{Understanding nuclear binding energy with single unified energy coefficient}

Interesting points to be noted are:

1. With reference to electromagnetic interaction, and based on proton number, $\left(1 / \alpha_{s}\right) \cong 8.68$ can be considered as the maximum strength of nuclear binding energy.

2. $\mathrm{Z} \approx 30$ seems to represent a characteristic reference number in understanding nuclear binding of light and heavy atomic nuclides.

Based on the new integrated model proposed by Ghahramany et al $[11,12]$,

$$
B(Z, N)=\left\{A-\left(\frac{\left(N^{2}-Z^{2}\right)+\delta(N-Z)}{3 Z}+3\right)\right\} \frac{m_{n} c^{2}}{\gamma}
$$

where, $\gamma=$ Adjusting coefficient $\approx(90$ to 100$)$.

if $N \neq Z, \delta(N-Z)=0$ and if $N=Z, \delta(N-Z)=1$.

Readers are encouraged to see references there in $[11,12]$ for derivation part. Point to be noted is that, close to the beta stability line, $\left[\frac{N^{2}-Z^{2}}{3 Z}\right]$ takes care of the combined effects of coulombic and asymmetric effects. In this context, we would like suggest that,

$$
\left.\begin{array}{l}
\frac{m_{n} c^{2}}{\gamma} \cong \frac{m_{n} c^{2}}{(90 \text { to } 100)} \cong \text { Constant } \\
\cong \frac{e_{s}^{2}}{8 \pi \varepsilon_{0}\left(G_{s} m_{p} / c^{2}\right)} \cong 10.09 \mathrm{MeV}
\end{array}\right\}
$$

Proceeding further, with reference to relation (19), it is also possible to show that, for $Z \cong(40$ to 83$)$, close to the beta stability line,

$$
\left[\frac{N_{s}^{2}-Z^{2}}{Z}\right] \cong k A_{s} Z
$$

Based on the above relations and close to the stable mass numbers of $(Z \approx 5$ to 118$)$, with a common energy coefficient of $10.09 \mathrm{MeV}$, we would like to suggest two terms for fitting and understanding nuclear binding energy.

First term helps in increasing the binding energy and can be considered as,

$$
\text { Term } \_1=A_{s} \times 10.09 \mathrm{MeV}
$$

Second term helps in decreasing the binding energy and can be considered as,

$$
\begin{gathered}
\text { Term_2 }=\left(\frac{k A_{s} Z}{2.531}+3.531\right) \times 10.09 \mathrm{MeV} \\
\text { where }\left\{\begin{array}{l}
\left(m_{n}-m_{p}\right) / m_{e} \cong 2.531 \\
3.531 \cong 1+\left[\left(m_{n}-m_{p}\right) / m_{e}\right]
\end{array}\right.
\end{gathered}
$$

Thus, binding energy can be fitted with,

$$
B_{A_{s}} \cong\left\{A_{s}-\left(\frac{k A_{s} Z}{2.531}+3.531\right)\right\} \times 10.09 \mathrm{MeV}
$$

See the following figure 1 . Dotted red curve plotted with relations (14) and (21) can be compared with the green curve plotted with the standard semi empirical mass formula (SEMF). For medium and heavy atomic nuclides, fit is excellent. It seems that some correction is required for light atoms.

Figure 1: Binding energy per nucleon close to stable mass numbers of $Z=5$ to 118 


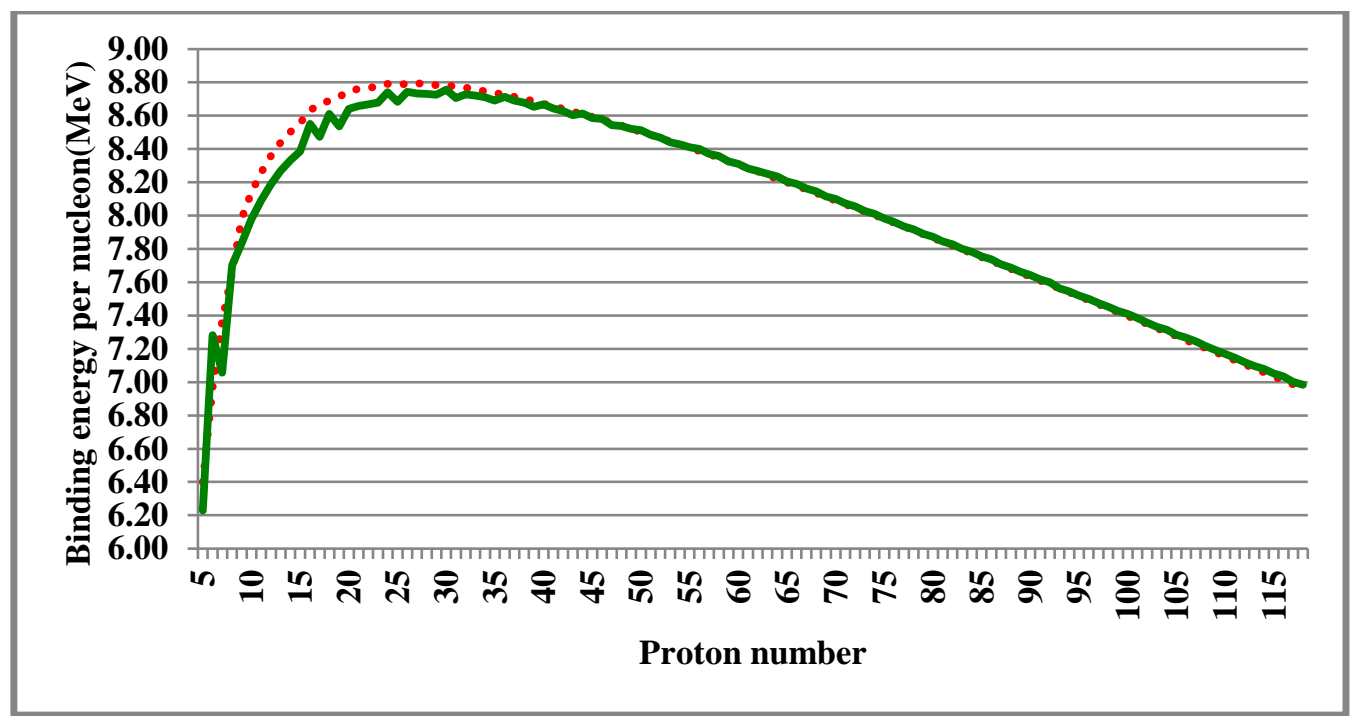

With trial and error, we have developed a third term of the form $\left[\left(A_{s}-A\right)^{2} / A_{s}\right] \times 10.09 \mathrm{MeV}$. Using this term, approximately, it is possible to fit the binding energy of isotopes in following way.

$$
B_{A} \cong\left\{\left[A-\left(\frac{k A Z}{2.531}+3.531\right)\right]-\left[\frac{\left(A_{s}-A\right)^{2}}{A_{s}}\right]\right\} \times 10.09 \mathrm{MeV}
$$

\section{Proton number}

See figure 2 for the estimated isotopic binding energy of $\mathrm{Z}=50$. Dotted red curve plotted with relations (14)

and (22) can be compared with the green curve plotted with SEMF. For $\mathrm{Z}=50$ and $\mathrm{A}=100$ to 130 , with reference SEMF, there is no much more difference in the estimation of binding energy. With reference to SEMF, when $(A>130)$, estimated binding energy seems to be increasing and when $(A \geq 212)$, estimated binding energy seems to be decreasing rapidly. It needs further study and refinement.

Figure 2: Binding energy of isotopes of $Z=50$

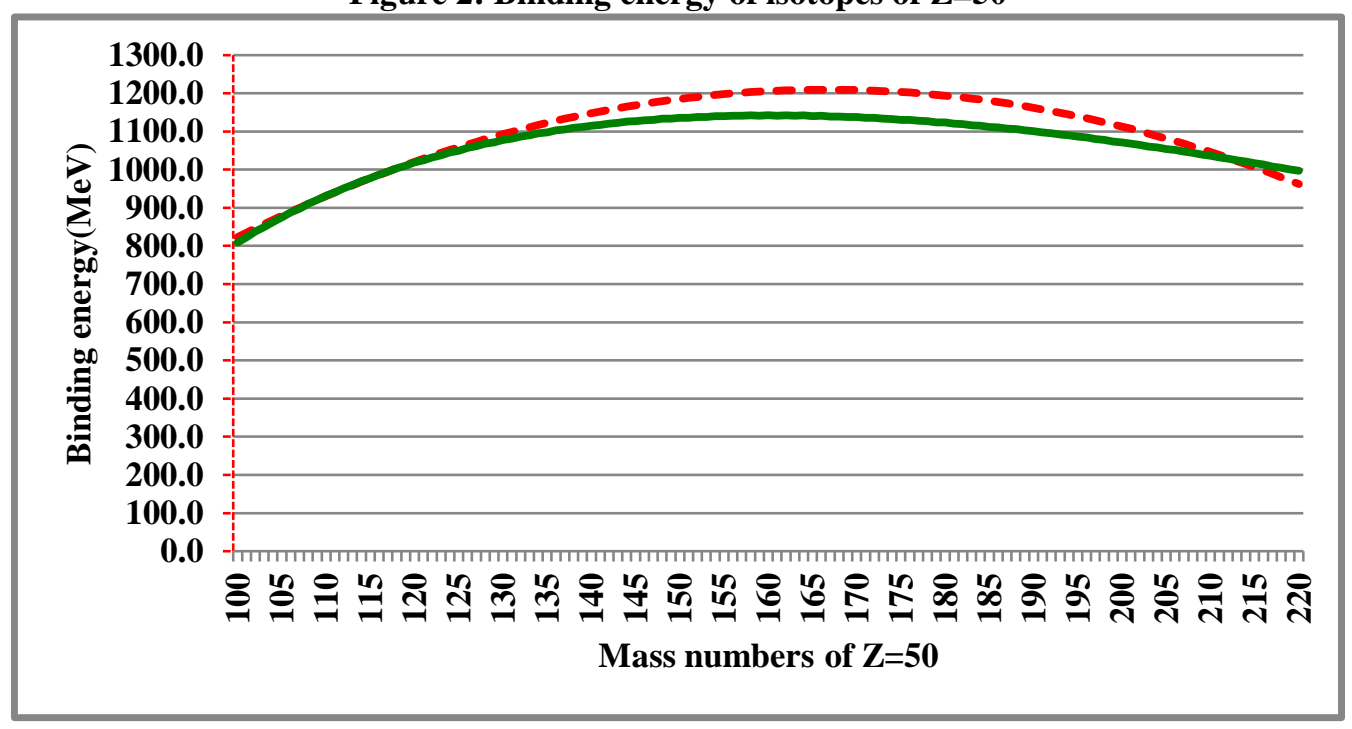

7. Understanding neutron life time with four gravitational constants 
One of the key objectives of any unified description is to simplify or eliminate the complicated issues of known physics. In this context, in a quantitative approach, we noticed that, the four gravitational constants play a crucial role in understanding and estimating neutron life time. The following three strange relations can be given some consideration [34].

$$
\begin{gathered}
\left(t_{n}\right)_{x} \approx\left(\frac{G_{e}}{G_{w}}\right)\left(\frac{G_{e} m_{n}^{2}}{\left(m_{n}-m_{p}\right) c^{3}}\right) \approx 874.94 \mathrm{sec} \\
\left(t_{n}\right)_{y} \approx \sqrt{\frac{G_{e}}{G_{N}}}\left(\frac{G_{s} m_{n}^{2}}{\left(m_{n}-m_{p}\right) c^{3}}\right) \approx 896.45 \mathrm{sec}
\end{gathered}
$$

Considering the geometric mean of relations (23) and (24), it is possible to show that,

$$
\left(t_{n}\right)_{x y} \approx \sqrt{\frac{G_{s}}{G_{w}} \sqrt{\frac{G_{e}}{G_{N}}}}\left(\frac{G_{e} m_{n}^{2}}{\left(m_{n}-m_{p}\right) c^{3}}\right) \approx 885.63 \mathrm{sec}
$$

Relation (25) seems to constitute all the four gravitational constants. It needs further study.

$$
\text { Considering } \quad\left(t_{n}\right)_{x}^{2 / 3}\left(t_{n}\right)_{y}^{1 / 3} \approx 882.05 \mathrm{sec} \quad \text { or }
$$

$\left(t_{n}\right)_{x}^{1 / 3}\left(t_{n}\right)_{y}^{2 / 3} \approx 889.2 \mathrm{sec}$, it may be possible to fit the experimental values of neutron life.

Plausible point to be noted is that, Relativistic mass of neutron seems to play a crucial role in understanding the increasing neutron life time. It can be understood with,

$$
t_{n} \propto \frac{m_{n}^{2}}{\left[1-\left(v^{2} / c^{2}\right)\right]}
$$

\section{Nuclear charge radii}

As per the current literature [35], nuclear charge radii can be expressed with the following formulae.

$$
\begin{aligned}
& R_{c} \cong\left\{1+\left[0.015\left(\frac{N-(N / Z)}{Z}\right)\right]\right\} Z^{1 / 3} \times 1.245 \mathrm{fm} \\
& R_{c} \cong\left\{1-0.349\left(\frac{N-Z}{N}\right)\right\} N^{1 / 3} \times 1.262 \mathrm{fm}
\end{aligned}
$$

$$
R_{c} \cong\left\{1-\left[0.182\left(\frac{N-Z}{A}\right)\right]+\frac{1.652}{A}\right\} A^{1 / 3} \times 0.966 \mathrm{fm}
$$

Our earlier proposed relation is,

$$
R_{(Z, A)} \cong\left\{Z^{1 / 3}+(\sqrt{Z(A-Z)})^{1 / 3}\right\}\left(\frac{G_{s} m_{p}}{c^{2}}\right)
$$

Based on these relations and by considering the charge radii of stable atomic nuclides, $R_{0}$ and $G_{s}$ can be fitted.

\section{Discussion}

1) At atomic and nuclear scales, so far there exist no generally accepted unified theoretical formulae or procedures for estimating the magnitude of Newtonian gravitational constant.

2) According to Rosi et al. [36] - There is no definitive relationship between $G_{N}$ and the other fundamental constants, and there is no theoretical prediction for its value, against which to test experimental results. Improving the precision with which we know $G_{N}$ has not only a pure metrological interest, but is also important because of the key role that $G_{N}$ has in theories of gravitation, cosmology, particle physics and astrophysics and in geophysical models.

3) In a unified approach, one can see the great initiative taken by John E. Brandenburg [37].

4) 2007 onwards, scientists and engineers are trying to estimate the magnitude of $G_{N}$ by 'Atomic interferometry' and gradiometers [38, 39, 40]. In this method, cold atoms are allowed to have free fall under gravity. Clearly speaking, an atomic gravity gradiometer is used to measure the differential acceleration experienced by two freely falling samples of laser-cooled rubidium atoms under the influence of nearby tungsten masses. Here we emphasize the point that, our approach is completely theoretical and no way connected with current experimental paradigm [41, 42] of $G_{N}$.

5) It is well established that, on large scales, universe is controlled by 'gravity' and on small scales, atoms are controlled by 'quantum mechanics'. In this context,

a) Based on the proposed relations (2) and (7), 


$$
\left.\begin{array}{l}
\hbar c \cong\left(\frac{m_{p}}{m_{e}}\right)^{2}\left(G_{e}^{2} G_{N}\right)^{1 / 3} m_{p}^{2} \\
\cong\left(\frac{e_{s} G_{s}}{e G_{w}}\right)\left(G_{e}^{2} G_{N}\right)^{1 / 3} m_{p}^{2}
\end{array}\right\}
$$

This relation seems to indicate a breakthrough in our understanding of quantum mechanics. As per current unification paradigm $\hbar c$ plays a key role in all the four interactions. Very interesting point to be noted is that, relation (30) constitutes all the four gravitational constants. It needs further study at basic level. We are working in this new direction.

b) With reference to electromagnetic and Newtonian gravitational constants, it is possible to show that,

Planck mass,

$$
M_{p l} \cong \sqrt{\frac{\hbar c}{G_{N}}} \cong\left(\frac{G_{e}}{G_{N}}\right)^{\frac{1}{3}}\left(\frac{m_{p}^{2}}{m_{e}}\right)
$$

c) With reference to nuclear and electromagnetic gravitational constants, it is possible to show that,

Bohr radius,

$a_{0} \cong\left(\frac{4 \pi \varepsilon_{0} G_{e} m_{e}^{2}}{e^{2}}\right)\left(\frac{G_{s} m_{p}}{c^{2}}\right) \cong 5.2918 \times 10^{-11} \mathrm{~m}$

Atomic radius,

$$
R_{\text {atom }} \cong\left(\frac{2 \sqrt{G_{s} G_{e}} m_{p}}{c^{2}}\right) \cong 33.1 \text { picometer }
$$

d) With reference to proposed nuclear elementary charge, nuclear and electromagnetic gravitational constants ,

$$
\begin{gathered}
\sqrt{\frac{e_{s}^{2}}{4 \pi \varepsilon_{0} G_{s} m_{p} m_{e}}} \cong 2 \pi \\
h c \cong \sqrt{\frac{e^{2} G_{s} m_{p}^{3}}{4 \pi \varepsilon_{0} m_{e}}} \cong \sqrt{\left(\frac{e_{s}^{2}}{4 \pi \varepsilon_{0}}\right)\left(G_{e} m_{e}^{2}\right)} \\
\hbar c \cong \sqrt{\left(G_{s} m_{p} m_{e}\right)\left(G_{e} m_{e}^{2}\right)}
\end{gathered}
$$

e) With reference to the nuclear gravitational constant and nuclear elementary charge,

Proton magnetic moment can be expressed with,

$$
\mu_{p} \cong \frac{e_{s} \hbar}{2 m_{p}} \cong \frac{e G_{s} m_{p}}{2 c} \cong 1.488142 \times 10^{-26} \mathrm{~J} / \mathrm{T}
$$

Neutron magnetic moment can be expressed with,

$$
\mu_{n} \cong \frac{\left(e_{s}-e\right) \hbar}{2 m_{n}} \cong 9.8171 \times 10^{-27} \mathrm{~J} / \mathrm{T}
$$

f) With reference to the three atomic gravitational constants, Bohr magneton can be expressed with,

$$
\mu_{B} \cong \frac{e \hbar}{2 m_{e}} \cong\left(\frac{G_{s}^{2}}{G_{e} G_{w}}\right)\left(\frac{e G_{e} m_{e}}{2 c}\right) \cong \frac{e G_{s}^{2} m_{e}}{2 G_{w} c}
$$

Based on relation (37),

$$
\mu_{B} \cong \frac{e \hbar}{2 m_{e}} \cong \frac{e \sqrt{\left(G_{s} m_{p}\right)\left(G_{e} m_{e}\right)}}{2 c}
$$

g) With reference to weak and Newtonian gravitational constants, it is possible to show that,

$$
\frac{G_{w}}{G_{N}} \cong\left(\frac{m_{p}}{m_{e}}\right)^{10}
$$

6) With reference to the proposed relations (15) to (22) connected with understanding the mechanism of nuclear stability and binding scheme, proposed three atomic gravitational constants can be validated.

7) With reference to the proposed relations (23) to (25) connected with neutron life time, proposed three atomic gravitational constants can also be validated.

\section{Conclusion}

As there is a large gap in between nuclear and Planck scales, with currently believed notion of unification paradigm, it seems impossible to implement gravity in atomic, nuclear and particle physics. Even though our approach is speculative, role played by the four gravitational constants seems to be natural. By implementing the four gravitational constants in String theory models, it may be possible to explore the hidden unified physics. With further study, a practical model of materialistic quantum gravity can be developed and magnitude of the Newtonian gravitational constant can be estimated in a theoretical approach bound to Fermi scale.

\section{Acknowledgements}


Author Seshavatharam is indebted to B. Gopi Srinivas, CEO, Ornnova India Pvt Ltd, Bangalore, India, professors shri M. Nagaphani Sarma, Chairman, shri K.V. Krishna Murthy, founder Chairman, Institute of Scientific Research in Vedas (I-SERVE), Hyderabad, India and Shri K.V.R.S. Murthy, former scientist IICT (CSIR), Govt. of India, Director, Research and Development, I-SERVE, for their valuable guidance and great support in developing this subject.

\section{References}

[1] K. Tennakone. Electron, muon, proton, and strong gravity. Phys. Rev. D 10, 1722 (1974).

[2] Sivaram, C, Sinha, K. Strong gravity, black holes, and hadrons. Physical Review D. 16 (6): 1975 1978. (1977).

[3] Salam, Abdus; Sivaram, C. Strong Gravity Approach to QCD and Confinement. Modern Physics Letters A, 8 (4): 321-326. (1993).

[4] C. Sivaram et al. Gravity of Accelerations on Quantum Scales. Preprint, arXiv:1402.5071 (2013)

[5] Roberto Onofrio. On weak interactions as shortdistance manifestations of gravity. Modern Physics Letters A 28, 1350022 (2013)

[6] O. F. Akinto, Farida Tahir. Strong Gravity Approach to QCD and General Relativity. arXiv:1606.06963v3 (2017)

[7] Weizsäcker, Carl Friedrich von, On the theory of nuclear masses; Journal of Physics 96 pages 431458 (1935)

[8] W. D. Myers et al. Table of Nuclear Masses according to the 1994 Thomas-Fermi Model.(from nsdssd.lbl.gov)

[9] P. Roy Chowdhury et al. Modified BetheWeizsacker mass formula with isotonic shift and new driplines. Mod.Phys.Lett. A20 1605-1618. (2005)

[10] J.A. Maruhn et al., Simple Models of ManyFermion Systems, Springer-Verlag Berlin Heidelberg 2010. Chapter 2, page:45-70.

[11] Ghahramany et al. New approach to nuclear binding energy in integrated nuclear model. Journal of Theoretical and Applied Physics 2012, $6: 3$

[12] N. Ghahramany et al. Stability and Mass Parabola in Integrated Nuclear Model. Universal Journal of Physics and Application 1(1): 18-25, (2013).

[13] Seshavatharam, U.V.S., \& Lakshminarayana S., Analytical estimation of the gravitational constant with atomic and nuclear physical constants. Proceedings of the DAE-BRNS Symp. on Nucl. Phys. 60, 850-851 (2015)
[14] Seshavatharam U. V. S, Lakshminarayana, S., A new approach to understand nuclear stability and binding energy. Proceedings of the DAE-BRNS Symp. On Nucl. Phys. 62, 106-107 (2017)

[15] Seshavatharam U. V. S, Lakshminarayana, S., On the Ratio of Nuclear Binding Energy \& Protons Kinetic Energy. Prespacetime Journal, Volume 6, Issue 3, pp. 247-255 (2015)

[16] Seshavatharam U. V. S, Lakshminarayana, S., Consideration on Nuclear Binding Energy Formula. Prespacetime Journal, Volume 6, Issue 1, pp.58-75 (2015)

[17] Seshavatharam U. V. S, Lakshminarayana, S., Simplified Form of the Semi-empirical Mass Formula. Prespacetime Journal, Volume 8, Issue 7, pp.881-810 (2017)

[18] Seshavatharam U. V. S, Lakshminarayana, S., On the role of strong coupling constant and nucleons in understanding nuclear stability and binding energy. Journal of Nuclear Sciences, Vol. 4, No.1, 7-18, (2017)

[19] Seshavatharam U. V. S, Lakshminarayana, S., A Review on Nuclear Binding Energy Connected with Strong Interaction. Prespacetime Journal, Volume 8, Issue 10, pp. 1255-1271 (2018)

[20]U. V. S. Seshavatharam, Lakshminarayana S. To unite nuclear and sub-nuclear strong interactions. International Journal of Physical Research, 5 (2) 104-108 (2017)

[21] Seshavatharam U.V.S \& Lakshminarayana S, A Virtual Model of Microscopic Quantum Gravity. Prespacetime Journal, Vol 9, Issue 1, pp. 58-82 (2018).

[22] Seshavatharam U.V.S \& Lakshminarayana S, To confirm the existence of nuclear gravitational constant, Open Science Journal of Modern Physics. 2(5): 89-102 (2015).

[23] Seshavatharam U.V.S \& Lakshminarayana S, Towards a workable model of final unification. International Journal of Mathematics and Physics 7, No1,117-130. (2016)

[24] U. V. S. Seshavatharam and S. Lakshminarayana. Understanding the basics of final unification with three gravitational constants associated with nuclear, electromagnetic and gravitational interactions. Journal of Nuclear Physics, Material Sciences, Radiation and Applications Vol-4, No1, 1-19. (2017)

[25] U. V. S. Seshavatharam et al. Understanding the constructional features of materialistic atoms in the light of strong nuclear gravitational coupling. Materials Today: 3/10PB, Proceedings 3 pp. 3976-3981 (2016)

[26] U. V. S. Seshavatharam, Lakshminarayana S. To Validate the Role of Electromagnetic and Strong Gravitational Constants via the Strong 
Elementary Charge. Universal Journal of Physics and Application 9(5): 210-219 (2015)

[27] U. V. S. Seshavatharam, Lakshminarayana S. Lakshminarayana. On the role of 'reciprocal' of the strong coupling constant in nuclear structure. Journal of Nuclear Sciences, Vol. 4, No.2, 31-44. (2017)

[28] Fermi scale applications of strong (nuclear) gravity-1 Proceedings of the DAE Symp. on Nucl. Phys. 63 72-73. (2018)

[29] Seshavatharam, U.V.S., \& Lakshminarayana S., On the Possible Existence of Strong Elementary Charge \& Its Applications. Prespacetime Journal, Volume 9, Issue 7, pp. 642-651 (2018)

[30] Seshavatharam U.V.S \& Lakshminarayana S. Scale Independent Workable Model of Final Unification. Universal Journal of Physics and Application 10(6): 198-206. (2016)

[31] C. Patrignani et al. (Particle Data Group), Chin. Phys. C, 40, 100001 (2016) and 2017 update

[32] Bethke and G.P. Salam. Quantum chromodynamics. K.A. Olive et al. (Particle Data Group), Chin. Phys. C, 38, 090001 (2014) and 2015 update.

[33] A. V. Karpov et al. Decay Properties and Stability of Heaviest Elements. International Journal of Modern Physics E Vol. 21, No. 21250013 (2012)

[34] RW Pattie Jr et al. Measurement of the neutron lifetime using a magneto-gravitational trap and in situ detection. Science 11, Vol. 360, Issue 6389, pp. 627-632 (2018)

[35] T. Bayram, S. Akkoyun, S. O. Kara and A. Sinan, New Parameters for Nuclear Charge Radius Formulas, Acta Physica Polonica B. 44(8), 17911799 (2013)

[36] G. Rosi, F. Sorrentino, L. Cacciapuoti, M. Prevedelli, and G. M. Tino. Precision measurement of the Newtonian gravitational constant using cold atoms, Nature 510 (7506), 518-521 (2014).

[37] John E. Brandenburg. The GEMS (Gravity-EM Super) Unification Theory: the Unification of the Four Forces of Nature, Prediction of New $21 \mathrm{MeV}$ and $22 \mathrm{MeV}$ Particles, and Correspondence with ElectroWeak Theory. Journal of Multidisciplinary Engineering Science Studies. Vol. 2 Issue 10, 968-984 (2016)

[38] G. Lamporesi et al. Determination of the Newtonian Gravitational Constant Using Atom Interferometry. Phys. Rev. Lett. 100, 050801 (2008)

[39] G. Rosi, Challenging the big $G$ measurement with atoms and light. J. Phys. B: At., Mol. Opt. Phys. 49(20), 202002 (2016)

[40] B. Canuel et al. Exploring gravity with the MIGA large scale atom interferometer. Science reports,
8:14064 (2018)

[41]C. Rothleitner and S. Schlamminger. Measurements of the Newtonian constant of gravitation, G. Rev. Sci. Instrum. 88, 111101 (2017)

[42] Li, Qing et al. Measurements of the gravitational constant using two independent methods. Nature 560, 582-588 (2018) 